\title{
Passive Microwave Radiometry (MWR) as a Component of Imaging Diagnostics in Ju- venile Idiopathic Arthritis (JIA)
}

\author{
A.V. Tarakanov ${ }^{1}$, E.S. Ladanova1 ${ }^{1}$, A.A. Lebedenko ${ }^{1}$, T.D. Tarakanova ${ }^{1}$, S. Vesnin ${ }^{5}$, I. \\ Goryanin $^{2,3,4}$
}

1. Rostov State Medical University, Rostov-on-Don, Russia.

2. Okinawa Institute Science and Technology, Japan

\section{University of Edinburgh, UK}

4. Institute Experimental and Theoretical Biophysics, RAS, Russia

\section{Medical Microwave Radiometry LTD., UK}

Corresponding author: Prof Igor Goryanin goryanin@gmail.com

\begin{abstract}
Juvenile idiopathic arthritis (JIA) is a disease with unknown causes within all forms of arthritis in children under 16 years of age. The diagnosis is made when other joint pathology is excluded. Difficulties in early and differential diagnosis lead to the rapid disability of patients and an unfavorable life prognosis. Therefore, timely diagnosis is necessary to prevent irreversible damage to the joints and preserve their function. Due to the widespread use of new technologies, modern multimodal imaging has gained recognition, which includes X-ray, ultrasound, and MRI. The combination of methods plays a key role in confirming the diagnosis, monitoring disease activity, prognosis during the course, and outcome in children with JIA. Each method has its own advantages and disadvantages. The introduction of the method of passive microwave radiometry (MWR), in combination with other imaging methods, makes it possible to expand the possibilities of screening the disease in the preclinical and early clinical phases.
\end{abstract}

Key words: juvenile idiopathic arthritis; X-Ray; ultrasound; MRI; passive microwave radiometry (MWR).

\section{Introduction}

Juvenile idiopathic arthritis (JIA) is a disease with no known cause that covers all forms of arthritis and lasts more than six weeks in children under 16 years of age. The diagnosis is made when other joint pathology is excluded. Due to the absence of a single pathognomonic laboratory or clinical sign, JIA is one of the exceptions that unites all forms of childhood arthritis, the etiology of which cannot be determined today. The disease is one of the most frequent and most disabling rheumatic diseases in children [1].

JIA is characterized by its high prevalence in populations. Among the population of Europe and North America, the frequency and prevalence range from 2-20 and from 16-150 cases per 100,000, 
respectively [2]. A noticeable difference in the frequency of JIA subtypes was noted in different geographic regions and/or ethnic groups. In Western countries, the most common subtype is oligoarthritis. At the same time, this category is rarely found in Costa Rica, India, New Zealand, and South Africa, where polyarthritis predominates [3,4]. In Asia, systemic arthritis is the cause of a large number of childhood arthritis, with reported incidences in India and Japan of up to $25 \%$ and $50 \%$, respectively [5,6]. On the territory of the Russian Federation, the prevalence of JIA reaches).062\% of the child population, with girls more likely to get sick [7].

JIA is difficult in early and differential diagnosis, which then leads to rapid disability, and for patients, an unfavorable life prognosis. Therefore, timely diagnosis and treatment are essential to prevent irreversible damage to joints and maintain their function. Clinical evidence supports a "window of opportunity" at an early stage of the disease. In this case, treatment induces higher rates of remission and improves long-term results [8].

The joints in children undergo drastic changes during the dynamic growth of the child. In this regard, it is necessary to differentiate pathological changes from physiological ones. Joint diseases in children are based on an idiopathic inflammatory process. It is believed that the disease has a multifactorial polygenic nature and the mechanisms of the disease (activation of the immune response) differ in different variants of the disease. JIA is currently regarded more as an autoinflammatory disease, based on the production of proinflammatory cytokines by activated $\mathrm{T}$ lymphocytes and macrophages with the development of chronic inflammation [7].

The inflammatory process begins in the synovial membrane of the joint with a violation of microcirculation and damage to the cells lining in the synovial membrane (the inner layer of the joint capsule). Synovitis occurs early in the natural history of arthritis. Inflammation is manifested by increased vascularization, capillary leaks, and joint effusion. Joint edema in rheumatoid arthritis reflects inflammation of the synovium and is realized both by congenital cells (e.g., monocytes, mast cells, and congenital lymphoid cells) and adaptive immune cells (e.g., T-helper cells and B-cells). The inflammatory process ultimately triggers an osteoclastic reaction that leads to bone erosion. They are an important pathological feature of rheumatoid arthritis and are associated with disease severity and functional outcome. Most erosions develop in the first two years of the disease and often occur within six months with an aggressive disease. Therefore, it is important to timely determine the degree of involvement of the joint in the inflammatory process using diagnostic methods [9]. 
The diagnosis of JIA is established based on anamnesis, physical examination, biochemical data. [10]. There are frequent complaints of pain in the bones and joints, swelling, and limitation of mobility. The most characteristic symptom of the disease is joint stiffness, which increases with the long stay in a certain position (sleep, prolonged sitting). However, this symptom is difficult to assess in young children. At the same time, the edema characteristic of arthritis is often not correctly defined by children and parents. Joint pain may be absent, especially in young children (peak incidence in children one-three-years-old). Therefore, pain is not necessary to make a diagnosis of JIA. In fact, if joint pain is the only complaint in the absence of stiffness, swelling, or activity restrictions, then the likelihood of arthritis is low $[11,12]$.

In the classic version, upon physical examination, active arthritis is characterized by edema, skin flushing, joints that are hot to the touch, and a decreased range of motion with pain. The differential diagnosis for suspected childhood arthritis is critical to prevent delayed diagnosis, complications, and predict the outcome and response to treatment.

Laboratory diagnostics can also help diagnose JIA. However, the absence of laboratory inflammatory markers in JIA (leukocytosis, increased platelet count, erythrocyte sedimentation rate and Creactive protein, anemia, hypoalbuminemia) does not exclude the diagnosis [13].

In the last 10-15 years, due to the widespread use of new technologies, modern multimodal imaging has gained recognition, which includes X-ray, ultrasound and MRI. This approach is becoming increasingly important and plays a key role in confirming the diagnosis, monitoring disease activity, and predicting the course and outcome in children with JIA. Imaging techniques are widely integrated into clinical practice to improve diagnosis, disease control, and outcome in children. For mutual understanding and comparative analysis of the results in many countries over the past decades, several international groups for the standardization and validation of various imaging methods have been created: American College of Rheumatology Pediatric Rheumatology Imaging Study Group (ACR); European League Against Rheumatism (EULAR) - Pediatric Rheumatology European Society (PRES); Pediatric Rheumatology European Society Imaging Working Party; The Norwegian Juvenile Idiopathic Arthritis Study; Childhood Arthritis and Rheumatology Research Alliance Ultrasound Group (CARRA) and others [14].

\section{X-Ray}

Conventional radiography remains an important diagnostic and monitoring tool in the treatment of arthritis. Currently, X-ray is the reference research method with a reasonable level of reproduci- 
bility [15]. Modern radiography is an inexpensive, readily available method that allows wide coverage of the affected areas. In addition, there are validated assessment methods and scoring systems that provided longitudinal comparisons in JIA [16].

The main disadvantage of radiography in pediatrics is that it uses ionizing radiation. Also, the method is not sufficiently sensitive in detecting early osteochondral changes and assessing the inflammatory process, as it only allows bone structure assessment while the pathological process begins in the synovial membrane. The destruction of the epiphyseal cartilage leads to persistent inflammation and hypertrophy of the synovial membrane. Only then do erosive changes after significant loss of cartilage become evident on radiographs [17]. Therefore, the value of the method is undeniable in the diagnosis of rheumatoid arthritis with the presence of erosions.

Radiographs are rarely useful in the diagnosis of JIA because, in the early stages, they are often normal, and the growth of bone tissue may occur earlier with the disease [18, 19]. For example, on radiographs, the presence of growth lines, or rather transverse lines migrating from the growth plate, may indicate underlying chronic inflammation. Although they are not specific for inflammation, they can manifest themselves with malnutrition, endocrinopathies, or fractures. In the early stages of the disease, narrowing of the joint spaces can be observed in 5\% of patients, erosion in $10 \%$, accelerated bone growth in $20 \%$ (more often with damage to the knee joint) [7]. Radiographs can also reveal a pattern of disease that suggests an alternative diagnosis such as gout, psoriasis, or chondrocalcinosis (pyrophosphate arthropathy). At the same time, a competent assessment of the X-ray picture of bones and joints in children, taking into account age and, probably, sex characteristics, will help reduce the number of errors in the work of a rheumatologist [15].

Thus, radiography plays an important but limited role in the early diagnosis of rheumatoid arthritis.

\section{Ultrasound}

Ultrasound in pediatric rheumatology plays an important role and is one of the most commonly used. The method is cost-effective, readily available, and non-radiating. It allows you to outline inflammatory and structural changes in arthritis, as well as to narrow the differential diagnosis, to determine the activity of the disease, to identify a subclinical disease, and monitor treatment and assess the severity of joint damage.

The criteria for evaluating the ultrasound data can be as follows: the amount of intra-articular effusion, the thickness of the synovial membrane, and articular hyaline cartilage. The most common results of ultrasound in arthritis are the establishment of inflammatory lesions of the peripheral joints, 
articular cavity (synovitis), tendon sheaths (tenosynovitis), detection of bursitis, enthesopathies, and erosions [20].

For example, in patients after arthroplasty, ultrasound is used to diagnose and monitor postoperative complications, which include abnormal accumulations of fluid in the area of the endoprosthesis, hematomas at various stages of resorption and organization, abscesses, tendon damage, neuropathies, for example, peroneal nerve palsy after the knee joint and sciatic nerve injury during hip arthroplasty [21]. However, the visual image in this method significantly depends on the anatomical and physiological characteristics of a particular patient, which presents particular difficulties in childhood, taking into account the characteristics of a growing organism [22]. So, for example, for younger age groups, there are no norm/pathology metric criteria for the thickness of the synovial membrane and cartilage. Ultrasound examination is significantly inferior in identifying central erosive changes located in the center of large joints, it cannot penetrate the bone due to the acoustic shadow from the overlying bone (some areas are inaccessible) and the low penetration of the ultrasound beam into the central part of the connection with high-frequency transducers. The limitations of the method also include the inability to investigate changes in the bone marrow that are associated with the active phase of the course [23].

In young children, where synovial tissue is difficult to distinguish from adjacent hypoechoic epiphyseal cartilage, assessment of the synovium is challenging. And in patients with an advanced stage of the disease, thickening of the synovial membrane and its vascularization may reflect so much little activity as restructuring against the background of long-term inflammation [22]. Doppler ultrasound analyzes synovial blood flow and can show the activity of the process; however, physiologically profuse vascularization of the epiphyseal cartilage must be distinguished from synovial hypervascularization associated with inflammation. A potential trap can be physiologically expressed vascularization of the synovial membrane and enthesis in healthy children, due to the differentiation of the osteoarticular system and abundant blood supply to the forming tissues [24].

According to Sudoł-Szopińska et al. 2017 [25] the disadvantages of ultrasound include the following: low specificity since several arthropathies exhibit the same spectrum of symptoms, inability to assess the state of the bone marrow, limited field of vision, significant dependence on expert experience, and quality of equipment, the need for special training.

To conduct a high-quality ultrasound scan, the doctor must be familiar with the changes occurring in a healthy developing joint; the change in the thickness of the epiphyseal cartilage should be compared with a healthy child of the same sex and age. According to Cherkasova 2019 [26] ultrasound is important in the onset of the disease because it allows: to determine the expansion of the 
joint spaces (effusion), uneven thickening and edema of the synovial membrane, hypervascularization of the synovial membrane, intra-articular effusion, erosion of the contours of the cortical layer of bones, uneven thickness and heterogeneity hyaline cartilage structure, and infiltration of soft tissues of varying severity.

Given the low interpretation of ultrasound data, it is necessary to conduct randomized controlled trials to evaluate not only the therapy received, but also new imaging technologies. In the case of remission in a significant number of cases, the residual disease cannot be differentiated from chronic arthritis. Criteria for remission in ultrasound examination, both in adults and in children with JIA, have not yet been established [25]. Another disadvantage of ultrasound is that it does not provide any information about the structure of the intramedullary bone. It is known that edematous bone marrow lesions detected by MR imaging are an important prognostic factor and are considered biomarkers of disease progression.

\section{MRI}

Due to its indisputable sensitivity in visualizing early signs of soft tissue inflammation and bone changes, MRI has several advantages: the absence of ionizing radiation, multiplanarity, and excellent contrast resolution of soft tissues, which make it possible to evaluate all joint structures. This is the only method that can objectify bone marrow. This is an indication for treatment to avoid irreversible destruction of the joint. But long-term studies are needed to distinguish bone marrow edema seen in healthy children versus bone marrow edema, which will develop before erosions in patients with JIA [24]. The clinical significance of bone marrow edema in children with JIA remains unclear, and due to its high prevalence in healthy children, it can be considered a sign of normal bone maturation.

It is difficult to differentiate between synovial tissue and intra-articular fluid on MRI. However, the "inflamed" synovium, in contrast to the intra-articular fluid, is enhanced by intravenous administration of paramagnetic Gd-containing contrast agents, which facilitates its recognition [27]. It is known that, on MRI, synovial thickening ( $\geq 2 \mathrm{~mm}$ ) that increases after contrast administration is considered an indicator of ongoing inflammation (synovitis), as it has been shown to be treatable. In addition, as recently published by Hemke et al. 2017 [28] The thickness of the synovial membrane in the knee joints of healthy children does not exceed $1.8 \mathrm{~mm}$. When clinical evaluation shows no signs of inflammation, but synovial thickening is observed on MRI, this is usually interpreted as subclinical synovitis. In JIA, it has previously been reported that synovial thickening on MRI is present in almost $50 \%$ of JIA patients who are considered clinically inactive. However, another explanation for the observed synovial thickening on MRI is persistent synovial change following chronic inflammation. Because of this controversy, the clinical significance of synovial thickening on MRI remains unclear 
[29]. According to the results of studies by E. Charlotte van Gulik, MRI in younger patients with JIA there may be more effective additional methods for monitoring disease activity. A significant proportion of children with clinically inactive JIA showed a thickening of the synovium on MRI (34.6\%). These results are consistent with previous studies of patients with JIA, which examined inconsistencies between clinical assessment and MRI findings [30, 31].

Since there are currently no long-term studies evaluating synovial thickening on MRI in children with clinically inactive JIA, it remains difficult to determine the cause(s) of the observed synovial thickening on MRI.

In the past few years, MRI has become more and more widely used in JIA, despite some limitations in its application: during one routine MRI examination, only one joint can be examined with all the required sequential contrast enhancements. However, this increases the likelihood of allergic reactions and stressful conditions in children.

There are also relative contraindications for MRI, such as claustrophobia, embedded metal fragments, pacemaker, and more [27]. MRI also has a high cost, limited availability, lack of standardized longitudinal and prospective studies, and, importantly, the need for sedation in young children (four to six years) for proper examination.

At the same time, indications for MRI have changed, shifting towards early detection of JIA. There has been an overall increased clinical demand for high-quality imaging. If multiple joints are affected, an MRI of all affected joints should be performed. These studies are time-consuming and expensive. Children need to lie still during the study, which is not very feasible for them; therefore, an alternative study is proposed. Currently, MRI of the whole body according to the protocol is widely used. Whole-body MRI is a diagnostic technique that allows you to obtain images of the entire body in just one scan. There is no standard protocol for examining full-body MRI in JIA. Various pulse sequences are often used. The examination can be done in coronal and sagittal or coronal and axial planes with or without contrast. Whole-body MRI can play an important role in assessing both active and asymptomatic joints for effusion, especially in areas that are not readily accessible to clinical methods (temporomandibular joint, sacroiliac joint) [24,32].

The main goal in JIA is to improve long-term results by early detection and treatment of disease activity, identification of children who are at risk of joint destruction, and poor functional outcome. This requires an accurate and sensitive determination of the diagnosis of synovitis, which is a sign of disease activity. Modern diagnostic imaging methods do not give a full-fledged answer to the assessment of the topic, onset, degree, and prognosis of the inflammatory process. In this regard, a broader multifactorial approach to diagnostics involving other methods is required. At the same time, as the 
analysis of the literature shows, the method should be informative, provide new data and not be burdensome for the child.

Analysis of the literature and our own research has identified a relatively new, but little developed method for JIA using passive microwave radiometry (MWR).

\section{MWR}

In recent years, the method of functional diagnostics has begun to spread widely, which consists in the dynamic mapping of physical fields and radiation from the human body, the method of microwave radiometry. It is based on measuring the intensity of passive thermal radiation, respectively, in the microwave (MWR) and infrared (IR) ranges. The spatial representation of the temperature distribution using MWR and IR inside and on the body's surface helps to determine the zone of the pathological process and, possibly, its qualitative properties [33, 34]. The essence of the biophysical noninvasive method of MWR is to measure the intrinsic electromagnetic radiation of internal tissues and to identify temperature anomalies of internal tissues of the body and skin [34]. The method for determining skin temperature has a long history. However, individual values of skin temperature fluctuate widely, which often leads to diagnostic errors. In recent years, methods have been developed for measuring the temperature distribution from the surface into the interior of the body, with the determination of the internal temperature profile $[35,36]$. The ambient temperature is of great methodological importance for the correct measurement of the depth temperature [37]. The method has a number of positive properties: no radiation exposure, the ability to carry out multiple measurements, non-invasiveness; high sensitivity, which allows detecting pathology at the preclinical stage; the simplicity and compactness of the equipment, as well as the clarity of the results are noted; containing no contraindications [38, 39].

The rationale for the use of MWR is the paradigm of temperature rise in inflammation. The classic sign of local inflammation is a rise in temperature. It is known that the development of chronic inflammation in JIA is mediated by various disorders in the immune system. It has been shown that the activity of inflammation correlates with changes in the synthesis of a significant spectrum of immune mediators. At the same time, cytokine proinflammatory cascades in different forms of the disease often differ [40]. The use of MWR in JIA involves the search for a local inflammatory process as a result of biochemical processes of local, complex vascular-mesenchymal tissue reactions in response to injury. The inflammatory process, including immunopathological, a priori, as a rule, has a 
higher temperature in comparison with the surrounding tissues. This does not depend on the etiological factor, but may depend on the time of the inflammation, the predominance of the exudative or proliferative process. The state of reactivity of the organism can also be a significant factor.

Systemic signs are recorded by clinical, biochemical, immunological and other methods. Temperature local changes, as a rule, precede structural changes. So, for example, at the stage of a clinically inactive state, it is possible to visually assess the presence or absence of an inflammatory process in the osteoarticular system (synovitis, tenosynovitis, enthesitis, etc.). JIA is characterized as a chronic non-suppurative inflammatory process of the synovial membranes. The morphological substrate of arthritis manifestations, especially associated with enthesitis, is an inflammatory fibrosing process with primary localization in tendon-ligamentous structures, their attachment points to the bone, as well as the synovial membrane of the joints with further lymphoid infiltration and hyperplasia [41, 42].

There are few publications on the use of MWR in JIA, and they are mainly in adult patients. The emergence of research depends significantly on new equipment and computers. For example, as early as 1987, it was shown that a strong correlation was found between the microwave thermographic index and the measured clinical and laboratory parameters when scanning 52 knees. This method has proven to be reproducible, fast, easy to use at the bedside without a controlled environment but has not been clinically followed up [43].

In recent years (2013-2019), a series of works by a group of authors (Zampeli, Raftakis, Laskari, Pentazos, Prekas, Tektonidou, and Siores et al.) has appeared on the use of RTM in joint diseases and rheumatoid arthritis in adults.

One of the first clinical studies was an article in which the concept that elevated local temperature reflecting subclinical inflammation of the synovium was tested using RTM. The ultrasound method acted as a reference method, as there was a possible presence of fluid and/or synovitis. The knees of healthy controls, patients with a recent knee injury, and patients without symptoms of rheumatoid arthritis or osteoarthritis were examined. Two settings of the radio antenna were used: a) in the upper third of the anterior surface of the thigh (control point) and b) above the patella. The main conclusion of the work was that thermal changes in the knee joint may reflect nonclinical pronounced joint inflammation [44].

In the next publication, the aim of the work was to establish a correlation between the depth temperature of small joints, obtained by the MWR method and parameters commonly used to assess disease activity in rheumatoid arthritis. Clinical and laboratory examinations, as well as ultrasound examination of joints and MWR of small joints of the hands and feet at baseline and 15, 30 and 90 days after the start of treatment were performed in 10 patients with active RA without treatment. 
Control subjectswere 20 healthy people of the same age and sex. We used 1248 individual RTM recordings from 16 patient joints. It has been established that an increased depth temperature obtained with the RTM, indicating local inflammation of small joints, can serve as an additional biomarker in RA [45].

After propaedeutic work on MWR, the authors studied 82 patients with rheumatoid arthritis and 23 healthy people of the same age and sex. Clinical and laboratory studies and ultrasound examinations of the joints were also carried out. Twenty-one patients were re-examined two months after the start of treatment. A decrease in temperature was found at points that were previously hotter $(\Delta \mathrm{t}$ is the difference between the temperature of predetermined joint points). The thermal score was created by summing up temperature values of the 7 small joints of the hand, as well as the elbow, knee, and lower leg. Clinically dominant upper/lower limb. The conclusion of the work was as follows: the elevated joint temperature detected by MWR is well suited for detecting inflammation of the knee joint in RA. The method makes it possible to distinguish between the stages of disease activity, as well as mirror changes in disease activity caused by treatment [46].

In their work, the team of authors (Laskari K. et al., 2019) suggested that an elevated joint temperature measured using a fast and easy-to-perform microwave radiometry method may reflect inflammation even in the absence of clinical signs. The group of patients $(n=243)$ included patients with rheumatoid arthritis and spondyloarthritis. Parallel studies using clinical examination, ultrasound examination of the joints and / or MRI have established that a deep increase in the temperature of the joints using MWR reflects both subclinical and clinically evident inflammation and can serve as a biomarker of arthritis [47].

Works on the application of the MWR method in pediatrics have since appeared. The RTM method was used to determine the depth and skin temperatures in the projection of the knee joints in healthy children aged 8-12 years for comparative analysis with patients. The study included girls (n $=22$, age $10.0 \pm 0.3$ years $)$ and boys $(n=21$, age $10.1 \pm 0.3$ years $)$. During the course of treatment, children from 6 to 17 years old $(n=43)$ who were in the clinic with a diagnosis of juvenile idiopathic arthritis were examined. The standards for the depth temperature in the knee joints in healthy children have been established, depending on the measurement zone. It is also shown that $\Delta \mathrm{T}=\mathrm{t} \max -\mathrm{t} \min$ of the depth temperature in the norm is $1.5-1.6^{\circ} \mathrm{C}$, and that of the skin, $1.4-1.7^{\circ} \mathrm{C}$. It was found that the temperature field of the skin in normal conditions practically repeats the pattern of the deep temperature field; the lowest temperature is noted above the patella. Clinical visual assessment of temperature data gives an instant idea of thermal asymmetry. MWRallows comparing the indicators of children with juvenile idiopathic arthritis (knee joints) with those of healthy children, assessing the 
thermal asymmetry of depth and skin temperatures by fields and fields of temperature difference, thermograms of depth, and skin temperatures [48].

Considering the described data, it is logical to combine MWR as an independent or adjuvant method for any imaging method, which will significantly expand the diagnostic capabilities. It is possible to determine the development of early inflammation and objectify the assessment of the severity of inflammatory processes in arthritis of various origins, including JIA. It is possible to control the effectiveness of the received basic therapy and timely cancellation if it is ineffective. At the same time, the lack of a sufficiently complete qualitative and quantitative description of the behavior of temperature fields in various human organs, both in the presence of pathology and in its absence, significantly complicates the development of effective methods of medical diagnostics. In our opinion, further work in this direction of easily interpreted imaging modalities can help pediatric and adult rheumatologists in joint assessment, especially if the clinical judgment is inconclusive. The correlation of MWR data with ultrasound, MRI, biochemical studies, immunological data, and clinic requires further study.

\section{References:}

1. Ravelli A., Handbook of Juvenile Idiopathic Arthritis, Springer International Publishing Switzerland. 2016 .-- 132 p. DOI 10.1007 / 978-3-319-08102-1_1

2. Ravelli, A. and Martini, A. (2007) Juvenile Idiopathic Arthritis. The Lancet, 369, 767-778. https://doi.org/10.1016/S0140-6736(07)60363-8

3. Petty RE, Cassidy JT. Chronic arthritis in childhood. In: Cassidy JT, Petty RE, Laxer RM, Lindsley CB, eds. Textbook of Pediatric Rheumatology. 6th edn. Philadelphia, PA: Elsevier Saunders; 2011; 211-235.

4. Szer IS, Kimura Y, Malleson PN, Southwood T, eds. Arthritis in Children and Adolescents. New York, NY: Oxford University Press; 2006.456 p.

5. Schwartz MM, Simpson P, Kerr KL, Jarvis JN. Juvenile rheumatoid arthritis in African Americans. J Rheumatol. 1997; 24 (9): 1826-1829.

6. Prakken, B., Albani, S. and Martini, A. (2011) Juvenile Idiopathic Arthritis. The Lancet, 377, 2138-2149.https: //doi.org/10.1016/S0140-6736 (11) 60244-4

7. Alekseeva E.I. Juvenile idiopathic arthritis: clinical presentation, diagnosis, treatment / Questions of modern pediatrics. 2015; 14 (1): 78-94). 
8. Malattia C., Rinaldi M., Martini A. (2018): The role of imaging in juvenile idiopathic arthritis, Expert Review of Clinical Immunology, DOI: 10.1080 / 1744666X.2018.1496019 / Jul 2018.

9. Teh J., Stergaard M. (2017) What the Rheumatologist Is Looking for and What the Radiolo-gist Should Know in Imaging for Rheumatoid Arthritis. Radiol Clin N Am - (2017): Sep; 55 (5): $905-$ 916. doi: 10.1016 / j.rcl.2017.04.001.

10. Torosyan GG, Zholobova ES, Glazyrina AA, Koltunov IE .. Difficulties in the diagnosis of systemic juvenile idiopathic arthritis. Pediatrics. 2016; 95 (5): 162-165.

11. Igisheva L.N., Pritchina S.S., Bykova Yu.A. Anikeenko A.A. Juvenile arthritis: diagnosis and treatment / Mother and child in Kuzbass. 2017. - No. 1 (68) .- S. 48-61.

12. Crayne C.B., Beukelman T. (2018): Juvenile Idiopathic Arthritis Oligoarthritis and Poly-arthritis, Pediatr Clin N Am 65 (2018) 657-674.

13. Union of Pediatricians of Russia, Juvenile arthritis: Clinical guidelines / Union of Pediatricians of Russia // Clinical guidelines. - 2017.- S. 8, 24-28.

14. Nusman C.M., Tanturri de Horatio L., Hemke R., E. Charlotte Van Gulik E.C. et.al. Imaging in juvenile idiopathic arthritis - international initiatives and ongoing work. 2018. - Pediatric Radi-ology https://doi.org/10.1007/s00247-017-4054-z

15. Kozhevnikov A.N., Pozdeeva N.A., Konev M.A., Maricheva O.N., Afonichev K.A., Novik G.A. X-ray diagnosis of juvenile chronic oligoarthritis. Bulletin of Siberian Medicine. 2017; 16 (3): 224-234. DOI 10.20538 / 1682-0363-2017-3-224-234

16. Teh J., Stergaard M. (2017) What the Rheumatologist Is Looking for and What the Radiolo-gist Should Know in Imaging for Rheumatoid Arthritis. Radiol Clin N Am - (2017): Sep; 55 (5): $905-$ 916. doi: 10.1016 / j.rcl.2017.04.001.

17. Hemke R., Tzaribachev N., Barendregt A.M., J. Merlijn van den Berg, Dori A.S., Maas M. (3 May 2018): Imaging of the knee in juvenile idiopathic arthritis, Pediatr Radiol

18. Courtney B. Crayne, Timothy Beukelman (2018): Juvenile Idiopathic Arthritis Oligoarthritis and Polyarthritis, Pediatr Clin N Am 65 (2018) 657-674.

19. Dimitriou Christos, Boitsios Grammatin, Phu-Quoc Lê, Laurence Goffin, Badot Valérie, PhDc, Paolo Simoni (2017): Imaging of Juvenile Idiopathic Arthritis, Radiol Clin N Am

20. Magni-Manzoni S. Pediatric Rheumatology / (2016) 14:33 DOI 10.1186 / s12969-016-0096-2 
21. Sudoł-Szopińska.I., Schueller-Weidekamm C., Plagou A., Teh J. (2017): Ultrasound in Arthritis. Radiol clinics of North America 55 (5): 985-996 DOI: 10.1016 / j.rcl. 2017.04.005

22. Alekseev D.L. Ultrasound examination of the musculoskeletal system in the diagnosis and monitoring of disease activity in juvenile arthritis / DL Alekseev, IP Nikishina // Scientific and Practical Rheumatology. -2017. No. 55 (6) - P. 647-654.

23. Traudt A.K. Magnetic resonance imaging in the diagnosis of juvenile arthritis / Traudt A.K., Zavadovskaya V.D., Zhogina T.V., Fedorova E.I.// Bulletin of Siberian Medicine.- 2015.-No. 1- P. 110-119

24. Christos D., Grammatin B., Lê Phu-Quoc, Goffin L., Valérie B., Simoni P. Imaging of Juvenile Idiopathic Arthritis, Radiol Clin N Am. - 2017.doi.org/10.1016/j.rcl.2017.04.011

25. Sudoł-Szopińska.I., Schueller-Weidekamm C., Plagou A., Teh J. (2017): Ultrasound in Arthritis. Radiol clinics of North America 55 (5): 985-996 DOI: 10.1016 / j.rcl. 2017.04.005

26. Cherkasova I.A. Radiation methods of research in the diagnosis of juvenile rheumatoid arthritis. Innovative medical technologies Sat. scientific papers. Ed. NS. Chirkova, S.V. Zhukov. Tver, Publisher: Tver Regional Public Movement to Support Young Scientists "Association of Young Scientists of the Tver Region" (Tver). 2019 .-- S. 77-79.

27. Reijnierse M., Van der Helm-Mil A., Iris Eshed I, Schueller-Weidekamm C., (2018) Magnetic Resonance Imaging of Rheumatoid Arthritis: Peripheral Joints and Spine. Semin Musculo-skelet Radiol 2018; 22 (02): 127-146. DOI: 10.1055 / s-0038-1639474

28. Hemke R, Berg J. M., Nusman C. M et al. (2017) Contrastenhanced MRI findings of the knee in healthy children; establishing normal values. Eur Radiol. https://doi.org/10.1007/s00330-017-50676

29. E. Van Gulik C., Welsink-Karssies M. M., Van den Berg Dieneke Schonenberg-Meinema J. M., Dolman K. M., Barendregt A. M., Nusman C. M., Maas1 M. , Taco W. Hemke K.R. (2018): Ju-venile idiopathic arthritis: magnetic resonance imaging of the clinically unaffected knee, Pediatric Radiology 48, pages 333-340 (2018) https://doi.org/10.1007/s00247-017-4059-7

30. Nusman C.M., Hemke R., Benninga M.A. et al. (2016) Contrastenhanced MRI of the knee in children unaffected by clinical arthritis compared to clinically active juvenile idiopathic arthritis patients. Eur Radiol 26: 1141-1148 
31. Nusman C. M., Hemke R., Schonenberg D. et al. (2014) Distribution pattern of MRI abnor-malities within the knee and wrist of juvenile idiopathic arthritis patients: signature of disease ac-tivity. AJR Am J Roentgenol 202: W439 - W446

32. Razinova A.A., Pozdnyakova O.F., Masalova V.V., Pozdnyakov A.V. et al. Magnetic resonance imaging according to the WHOLE BODY protocol in children: clinical application (literature review) / Visualization in medicine. 2020. - No. 1 (2). - S. 3-15.

33. Vaysblat A.V. Radiothermography as a diagnostic method in medicine / A.V. Weisblat. M.: $2003 .--67$ p.

34. Vaysblat A.V. Medical radiothermometer RTM-01-RES // J. Biomedical technologies and radio electronics. - 2001. - No. 8. - P.11-23.

35. Losev A.G., Khoperskov A.V., Astakhov A.S., Suleimanova H.M. Problems of measurement and modeling of thermal and radiation fields in biological tissues: analysis of microwave thermometry data Vestn. Volgogr. state un-that. Ser. 1, Mat. Phys. 2015. No. 6 (31). - S. 31-71.

36. Goryanin I., Karbainov S., Shevelev O., Tarakanov A., Redpath K., Vesnin S., and Ivanov Y. Passive microwave radiometry in biomedical studies / Drug Discov Today 202004 28; 25 (4): 757 763. https://doi.org/10.1016/j.drudis.2020.01.016

37. Tarakanov A.V., Tarakanov A.A., Vesnin S., Efremov V.V., Roberts N. and Goryanin I. Influence of ambient temperature on recording of skin and deep tissue temperature in region of lum-bar spine / Eur J Mol Clin Med. - 2020. - No. 7 (1). - P. 21-26. DOI: https://doi.org/10.5334/ejmcm.274

38. A. V. Tarakanov. Microwave radiometry for pain in the lumbar spine. Application prospects. Methods for assessing and diagnosing chronic pain / A.V. Tarakanov, V.V. Efremov, A.A. Cockroaches // Russian Journal of Pain. - 2016. - No. 2 - S. 113-114.

39. Tarakanov AV, Tarakanov AA, S. Vesnin S., Efremov VV, Goryanin I., Roberts N. Mi-crowave Radiometry (MWR) temperature measurement is related to symptom severity in patients with Low Back Pain (LBP) / Journal of Bodywork \& Movement Therapies 26 (2021) 548e552. https://doi.org/10.1016/j.jbmt.2021.02.005

40. Chernyshova O.E., Konyushevskaya A.A., Vayzer N.V., Balychevtseva I.V. Juvenile arthritis: etiology, pathogenesis, modern aspects (literature review) / Trauma. 2018. - No. 2 (19). - S. 99-105. 41. Serebrennikova S.N., Semensky I.Zh. Pathophysiology of the inflammatory process. Tutorial. Irkutsk, ISMU, 2014 .-- 73 p. 
42. Alekseeva E.I., Litvitsky P.F. Juvenile rheumatoid arthritis: etiology, pathogenesis, clinic, diagnostic and treatment algorithm: hands. for doctors, teachers, scientific. sotr. / Under the general editorship of A.A. Baranov. - M.: VEDI, 2007 .-- 368 p.

43. Fraser, S.M., Land, D.V., Sturrock, R.D. Microwave thermography in rheumatic disease. MEP Ltd. 1987, 16 (4), 209-212. Doi: 10.1093 / rheumatology / 26.1.37)

44. Zampeli E, Raftakis I, Michelongona A, Nikolaou C, Elezoglou A, et al. (2013) Detection of Subclinical Synovial Inflammation by Microwave Radiometry. PLoS ONE 8 (5): e64606. doi: 10.1371 / journal.pone.0064606

45. Pentazos G., Laskari K., Prekas K., Raftakis J, Sfikakis PP, Elias Siores E. Microwave Ra-diometry-Derived Thermal Changes of Small Joints as Additional Potential Biomarker in Rheumatoid Arthritis: A Prospective Pilot Study / - J Clin Rheumatol. 2018 Aug; 24 (5): 259-263. doi: 10.1097 / RHU.0000000000000719.

46. Laskari K, Pentazos G, Konstantonis G, Tektonidou M, Siores E, Sfikakis P. Microwave Radiometry As a Novel Additional Method for Rheumatoid Arthritis Disease Activity Assessment: A Prospective Single-Center Study [abstract]. Arthritis Rheumatol. 2018; 70 (suppl 10). https://acrabstracts.org/abstract/microwave-radiometry-as-a-novel-additional-method-for- rheuma-toid-arthritisdisease-activity-assessment-a-prospective-single-center-study /. Accessed November 13, 2019.

47. Laskari1 K., Pentazos G., Pitsilka D., Raftakis J., Konstantonis G., Toutouzas K „, Siores E., Tektonidou M. and Sfikakis P. Joint radiometry for inflammatory arthritis assessment / September 2019 Rheumatology 59 ( 4) doi: 10.1093 / rheumatology / kez373

48. Tarakanov A.V., Lebedenko A.A., Ladanova E.S., Tarakanova T.D. Prospects for the use of microwave radiothermometry of the knee joints in children with juvenile idiopathic arthritis / Modern problems of science and education. - 2020. - No. 5; DOI 10.17513 / spno.30099 